\title{
A NOTE ON THE DIFFERENCES OF THE CONSECUTIVE POWERS OF OPERATORS
}

\author{
ANDRZEJ ŚWIȨCH \\ School of Mathematics, Georgia Institute of Technology \\ Atlanta, Georgia 30332, U.S.A. \\ E-mail: swiech@math.gatech.edu
}

\begin{abstract}
We present two examples. One of an operator $T$ such that $\left\{T^{n}(T-I)\right\}_{n=1}^{\infty}$ is precompact in the operator norm and the spectrum of $T$ on the unit circle consists of an infinite number of points accumulating at 1 , and the other of an operator $T$ such that $\left\{T^{n}(T-I)\right\}_{n=1}^{\infty}$ is convergent to zero but $T$ is not power bounded.
\end{abstract}

Let $\mathcal{A}$ be a Banach algebra and $x \in \mathcal{A}$ be power bounded. Denote by $\Gamma$ the unit circle in $\mathbb{C}$. The main aim of this note which may be regarded as an addendum to [2] is to answer a question stated there if the precompactness of $\left\{x^{n}(x-1)\right\}_{n=1}^{\infty}$ in $\mathcal{A}$ implies that $1 \notin \overline{\sigma(x) \cap(\Gamma \backslash\{1\})}$. The structure of $\sigma(x)$ in this case has been investigated in [1] and [2] (see the references quoted therein). It was proved in [1] that $\left\{x^{n}(x-1)\right\}_{n=1}^{\infty}$ is precompact if and only if $\sigma(x) \cap(\Gamma \backslash\{1\})$ consists of simple poles of $x$. The example that we present below shows that 1 can belong to the closure of $\sigma(x) \cap(\Gamma \backslash\{1\})$ and therefore the result quoted above is sharp.

Example 1. Let $\lambda_{n}=e^{2 \pi i / n}$. Define $T: l^{2} \rightarrow l^{2}$ by $T e_{n}=\lambda_{n} e_{n}, n=1,2, \ldots$, where $e_{n}=(0, \ldots, 0,1,0, \ldots)$ is the $n$th standard basis vector of $l^{2}$. Then $\|T\|=1$ and $\sigma(T)=\left\{\lambda_{1}, \lambda_{2}, \ldots\right\}$. We claim that $\left\{T^{n}(T-I)\right\}_{n=1}^{\infty}$ is precompact in the algebra of bounded operators on $l^{2}$ equipped with the operator norm. This fact can be deduced from the above quoted result of [1], however we will give a simple and direct proof. We will show that for every increasing sequence $\left\{n_{k}\right\}_{k=1}^{\infty}$ of positive integers we can choose a subsequence $\left\{m_{k}\right\}_{k=1}^{\infty}$ such that $T^{m_{k}}(T-I)$ is convergent as $k \rightarrow \infty$. We construct $\left\{m_{k}\right\}_{k=1}^{\infty}$ as follows. Since $\lambda_{2}^{n_{k}}$ attains only a finite number of values we choose a subsequence $\left\{n_{2_{k}}\right\}_{k=1}^{\infty}$ such that $\lambda_{2}^{n_{2_{k}}}=\bar{\lambda}_{2}$ for some $\bar{\lambda}_{2}$. Then, since $\lambda_{3}^{n_{2_{k}}}$ takes on only a finite number of values, we choose from $\left\{n_{2_{k}}\right\}_{k=1}^{\infty}$ a subsequence $\left\{n_{3_{k}}\right\}_{k=1}^{\infty}$ such that $\lambda_{3}^{n_{3}}=\bar{\lambda}_{3}$ for some $\bar{\lambda}_{3}$. We continue this process and define $m_{1}=n_{1}$ and $m_{k}=n_{k_{k}}$ for

1991 Mathematics Subject Classification: Primary 46H05, 46H30, $46 \mathrm{H} 35$.

The paper is in final form and no version of it will be published elsewhere. 
$k>1$. For $m_{k}$ so defined we have

$$
\lambda_{j}^{m_{k}}=\bar{\lambda}_{j} \quad \text { for } k \geq j, k, j=1,2, \ldots
$$

Notice that $m_{k} \geq k$ for $k=1,2, \ldots$ Let $\varepsilon>0$. Choose $n_{0}$ such that $\left|\lambda_{n}-1\right|<\varepsilon / 2$ for $n \geq n_{0}$. Denote by $P$ the orthogonal projection onto $\overline{\operatorname{span}}\left\{e_{n_{0}+1}, e_{n_{0}+2}, \ldots\right\}$. Take any $k, l \geq n_{0}$. By the definition of $T$ and (1) it follows that

$$
\left\|\left(T^{m_{k}}-T^{m_{l}}\right)(T-I) x\right\|=\left\|\left(T^{m_{k}}-T^{m_{l}}\right)(T-I) P x\right\| \leq 2\|(T-I) P\|\|x\| \leq \varepsilon\|x\|,
$$

where the last inequality follows from the choice of $n_{0}$. Therefore $\left\{T^{m_{k}}(T-I)\right\}_{k=1}^{\infty}$ is Cauchy. It converges to $T_{0}$ defined by $T_{0} e_{n}=\bar{\lambda}_{n} e_{n}, n=1,2, \ldots$ The claim is proved.

An interesting feature of Example 1 is that the set $\left\{T^{n}\right\}_{n=1}^{\infty}$ is discrete. In fact, if $n-m=p>0$, then

$$
\left\|\left(T^{n}-T^{m}\right) e_{2 p}\right\|=\left\|2 e^{\pi m i / p} e_{2 p}\right\|=2 .
$$

However, if we set $\lambda_{n}=e^{\frac{2 \pi i}{2^{2}}}, n=1,2, \ldots$, in the definition of $T$, then $\left\{T^{n}\right\}_{n=1}^{\infty}$ has an accumulation point. To show this observe that, if $x=\sum_{n=1}^{\infty} x_{n} e_{n},\|x\| \leq 1, p_{n}=2^{n^{2}}$, then

$$
\begin{aligned}
\left\|\left(T^{p_{n}}-I\right) x\right\| & =\left\|\sum_{j=n+1}^{\infty}\left(\lambda_{j}^{p_{j}}-1\right) x_{j} e_{j}\right\|=\left(\sum_{j=n+1}^{\infty}\left|\lambda_{j}^{p_{j}}-1\right|^{2}\left|x_{j}\right|^{2}\right)^{1 / 2} \\
& \leq\left(\sum_{j=n+1}^{\infty}\left(\arg \left(\lambda_{j}^{p_{j}}\right)\right)^{2}\right)^{1 / 2} \leq\left(\sum_{j=n+1}^{\infty} \frac{16 \pi^{2}}{2^{4 j}}\right)^{1 / 2} \\
& =\frac{4 \pi}{\sqrt{15}} \frac{1}{2^{2 n}} \rightarrow 0 \quad \text { as } n \rightarrow \infty
\end{aligned}
$$

Therefore the existence of accumulation points depends also on the geometry of the spectrum and not only on its cardinality. Moreover, it trivially follows that if $\left\{x^{n}\right\}_{n=1}^{\infty}$ has an accumulation point then $\sigma(x)=\sigma_{1} \cup \sigma_{2}$, where $\sigma_{1}, \sigma_{2}$ are two closed sets such that $\sigma_{1} \subset\{z:|z|<1\}, \sigma_{2} \subset \Gamma$. To see this, suppose that $x^{n_{k}}$ converges as $k \rightarrow \infty$ for some sequence $\left\{n_{k}\right\}_{k=1}^{\infty}$, i.e. for every $\varepsilon>0$ there is $n_{0}$ such that $\left\|x^{n_{k_{1}}}-x^{n_{k_{2}}}\right\|<\varepsilon$ if $n_{k_{1}}, n_{k_{2}}>n_{0}$. Let $n_{k_{2}}>n_{k_{1}}$. We have $\sigma\left(x^{n_{k_{1}}}-x^{n_{k_{2}}}\right)=\left(z^{n_{k_{1}}}-z^{n_{k_{2}}}\right)(\sigma(x))$. However,

$$
\left|z^{n_{k_{1}}}-z^{n_{k_{2}}}\right| \geq|z|^{n_{k_{1}}}-|z|^{n_{k_{2}}}
$$

and if we fix $n_{k_{1}}$, the right hand side of (2) can be made arbitrarily close to 1 by choosing $|z|$ sufficiently close to 1 and then $n_{k_{2}}$ sufficiently large. Hence $\sigma(x)$ cannot approach $\Gamma$.

We would like to finish with an elementary example of an operator $T$ such that $T^{n}(T-I) \rightarrow 0$ as $n \rightarrow \infty$ but $T$ is not power bounded. It is similar in the spirit to Example 3.7 in [2], ours is however very explicit.

ExAmple 2. Let $T: l^{2} \rightarrow l^{2}$ be defined by

$$
T e_{2 n}=\frac{n-1}{n} e_{2 n}+\frac{1}{\ln (n+1)} e_{2 n-1}, \quad T e_{2 n-1}=e_{2 n-1}
$$


for $n=1,2, \ldots$ Let $\varepsilon>0, x=\sum_{n=1}^{\infty} x_{n} e_{n}$. Then

$$
\begin{aligned}
\left\|\left(T^{k+1}-T^{k}\right) x\right\|^{2}= & \left\|\sum_{n=1}^{\infty}\left(\frac{1}{\ln (n+1)}\left(\frac{n-1}{n}\right)^{k} x_{2 n} e_{2 n-1}-\frac{1}{n}\left(\frac{n-1}{n}\right)^{k} x_{2 n} e_{2 n}\right)\right\|^{2} \\
\leq & \sum_{n=1}^{\infty} \frac{1}{\ln ^{2}(n+1)}\left(\frac{n-1}{n}\right)^{2 k}\left|x_{2 n}\right|^{2}+\sum_{n=1}^{\infty} \frac{1}{n^{2}}\left(\frac{n-1}{n}\right)^{2 k}\left|x_{2 n}\right|^{2} \\
\leq & \sum_{n=1}^{n_{0}} \frac{1}{\ln ^{2}(n+1)}\left(\frac{n-1}{n}\right)^{2 k}\left|x_{2 n}\right|^{2}+\frac{1}{\ln ^{2}\left(n_{0}+1\right)} \sum_{n=n_{0}+1}^{\infty}\left|x_{2 n}\right|^{2} \\
& +\sum_{n=1}^{n_{0}} \frac{1}{n^{2}}\left(\frac{n-1}{n}\right)^{2 k}\left|x_{2 n}\right|^{2}+\frac{1}{\left(n_{0}+1\right)^{2}} \sum_{n=n_{0}+1}^{\infty}\left|x_{2 n}\right|^{2} \leq \varepsilon^{2}\|x\|^{2}
\end{aligned}
$$

by first choosing $n_{0}$ sufficiently large and then taking $k$ large enough. To see that $T$ is not power bounded, observe that

$$
\left\|T^{n+1} e_{2 n}\right\| \geq \frac{1}{\ln (n+1)} \sum_{j=1}^{n}\left(\frac{n-1}{n}\right)^{j} .
$$

Since $\left(\frac{n-1}{n}\right)^{n}>\frac{1}{3}$ for large $n$ (actually it is close to $1 / e$ ), we obtain

$$
\left\|T^{n+1} e_{2 n}\right\| \geq \frac{n}{3 \ln (n+1)} .
$$

Hence $\left\|T^{n}\right\| \geq O\left(n^{\alpha}\right)$ for every $0<\alpha<1$. On the other hand, we have $T^{n}=o(n)$.

\section{References}

[1] S. Huang, Stability properties characterizing the spectra of operators on Banach spaces, J. Funct. Anal. 132 (1995), 361-382.

[2] H. C. Rönnefarth, On the differences of the consecutive powers of Banach algebra elements, this volume, 297-314. 